\title{
The action dynamics of overcoming the truth
}

\author{
Nicholas D. Duran, Rick Dale, and Danielle S. McNamara \\ University of Memphis, Memphis, Tennessee
}

\begin{abstract}
A convincing deceiver must act in discordance with their knowledge of the truth. To do so requires the deceiver to resolve competition between what is known to be true and what is intended to be false. We investigated the temporal signature of this competition by examining the action dynamics of arm movement while participants responded falsely or truthfully to autobiographical information. The participants answered no or yes by navigating a Nintendo Wii Remote to NO and YES regions on a large projector screen. Trajectory analyses of the fine-grained arm movements show increased complexity in false responding relative to truthful responding, with the greatest difference in false yes answers. The dynamic motor movements also reveal greater strength of competition during the act of false responding, thereby extending traditional response time measures that capture latent competition alone. These results suggest that deceptive processes may be detectable when action is allowed to covary with thought. Supplemental figures and a list of the sentence stimuli may be downloaded from http://pbr.psychonomic-journals.org/content/supplemental.
\end{abstract}

Most people can easily confirm or deny an assertion that they once met Elvis Presley or that they collect clocks in their spare time. Nonetheless, evidence suggests that prior to a final confirmation or denial of such assertions, people temporarily and perhaps nonconsciously believe the assertions to be true (Gilbert, 1991; Gilbert, Tafarodi, \& Malone, 1993). The nature of belief appears to be biased toward initially accepting a proposition as true, even when that proposition is unequivocally false. It stands to reason that for someone to dishonestly confirm or disconfirm any proposition, as in agreeing that they met Elvis when they really did not, the person will accept the assertion as true, then assess whether their initial acceptance is correct (which it is not), and then respond in order to violate the conclusion of their assessment (by falsely responding yes). In such deceptive behavior, competition exists between the initial belief, the assessment of the belief, and the intention to deceive. Although the initial belief is involuntary, this acceptance of the truth is an impediment that requires active processing to overcome.

By many accounts of deception, the competition and resolution involved in false responding is a far more challenging and time-consuming process than confirming the truth (Vendemia \& Buzan, 2004; Walczyk et al., 2005). Accordingly, researchers interested in deception detection have designed clever tasks that exploit this increased processing time (e.g., Gregg, 2007; Sartori, Agosta, Zogmaister, Ferrara, \& Castiello, 2008). Participants often respond honestly or deceptively to simple statements, and the time taken to respond, by vocal onset or a manual keypress, is recorded and analyzed. In general, these response time latencies are useful in discriminating certain deceptive behaviors and have thus risen to prominence as a standard bearer of detecting deception. Unfortunately, response time captures only the outcome of a completed cognitive process, and the real-time cognitive dynamics that occur during the process are lost.

To begin exploring these moment-to-moment changes of response selection, we turn to a growing body of action dynamics research. In this research, actions that occur in conjunction with a cognitive task often reflect ongoing characteristics of processing, ranging from low-level speech perception (Spivey, Grosjean, \& Knoblich, 2005) to higher level learning (Dale, Roche, Snyder, \& McCall, 2008). The response activity involved in these tasks is usually recorded as arm movements within a set spatial region. Analysis of the arm movement provides insight into what information is important during processing and when that information is most relevant. For example, in Dale, Kehoe, and Spivey (2007), participants used a computer mouse to make typicality judgments on category membership. Each trial involved matching an animal exemplar (e.g., whale) to one of two visually copresent response options located in opposite corners of a computer screen. For some trials, classification was potentially ambiguous, such as matching whale to a fish or mammal option. During response movements, the streaming $x, y$ coordinates of the computer mouse were recorded. Dale et al. (2007) found that computer mouse trajectories for atypical animals (e.g., whale) curved more toward a featurally similar distractor (e.g., fish), suggesting that semantic categorization processes also unfold partly into the dynamics of response execution.

We use this cognition-action interplay to tap the dynamics of false responding. Indeed, there is well-established evidence that deception often leaks into a deceiver's actions, such as with gesture and body posture (Vrij, 2001).

N. D. Duran, nduran@memphis.edu 
Here, we employed an action dynamics technique to study response behavior as continuous competition from an initial belief and the goals of deception. To the extent that this competition is expressed in action, we can use $x, y$ coordinate trajectories to expose the dynamics of overcoming this initial true belief and enacting the agenda of a false response.

In the present study, we expose hidden cognitive activity that is involved when one falsely accepts or denies assertions about oneself. To do so, we use a guided lie paradigm that is commonly employed in EEG and fMRI analysis (e.g., Spence et al., 2004; Vendemia \& Buzan, 2004). In our version, participants are prompted to respond falsely or truthfully to simple autobiographical facts, such as Have you ever been to Asia? Here, rather than answering with a computer keypress, the participants used a Nintendo Wii Remote, and the $x, y$ coordinates of their arm movements were rapidly sampled (see Dale et al., 2008).

With this rich data output, we evaluated signatures of deception in terms of the shape of each movement trajectory and the location of the trajectory over time. We also quantified trajectory properties on dimensions of velocity, stability, and direction. As the results reveal, the unpacking of response time provides not only unique distinctions between false and true responses, but also the more subtle distinction between false responses answered with either a no or a yes.

\section{EXPERIMENT Revealing the Dynamics of False and True Responding}

\section{Method}

Participants. Twenty-six undergraduate students (19 female, 7 male) participated for extra credit. Only native English speakers with normal or corrected-to-normal vision were eligible to participate. All of the participants were right-hand dominant.

Procedure. A trial began with a small bull's-eye-shaped circle appearing at the bottom center region of a $3.8 \times 1.8 \mathrm{~m}$ screen positioned approximately $2.7 \mathrm{~m}$ directly in front of the participant. The participants' task was to click on the circle with the Wii Remote-controlled cursor, and by their doing so, the first word in a biographical question would appear above the circle. With each click of the Wii Remote, the current word was replaced with the next word in the question. This process continued until the final word of the question was encountered (akin to self-paced reading tasks; Just, Carpenter, \& Woolley, 1982). At this point, a NO response box appeared in the top right corner of the screen, and a YES response box appeared in the top left corner of the screen (each box was approximately $0.5 \times 0.5 \mathrm{~m}$ ). Also at this time, the bull's-eye-shaped circle changed to green or red. If the circle changed to green, the participants were simply instructed to answer the question truthfully. If the circle changed to red, the participants were to answer falsely. All responses were made by navigating the Wii Remote cursor to the appropriate NO or YES box.

During each trial, the $x, y$ coordinates of the cursor movement were continuously recorded (sampling at approximately $80 \mathrm{~Hz}$ ) and stored for later processing. Because the Wii Remote was held with an extended arm in front of the body and toward the screen, subtle directional changes in wrist and arm movement were captured, with 33 pixels traversed for each centimeter of lateral movement.

The participants responded to 84 question trials, including 4 practice trials. The 80 experimental trials were divided equally between false and true color prompts, and the order of the false and true prompts and the order of the questions were randomized for each participant. The position of the NO and YES response boxes was also reversed for a third of the participants (i.e., the NO response box appeared in the top left corner of the screen, and the YES response box appeared in the top right corner of the screen). After the initial set of questions had been answered, the participants completed a followup verification task that required them to truthfully reanswer all of the questions that they had previously viewed.

Question stimuli. The questions used in the study began with the stem Have you ever . . . and were completed with 120 possible statements (i.e., Have you ever eaten pizza?; the stimuli are available online ${ }^{1}$ ). Eighty of the 120 questions were randomly selected for each participant. The questions were also selected to elicit an equal number of false no responses, true no responses, false yes responses, and false no responses. A pilot study confirmed that the responses were approximately evenly distributed within individuals across our target population. All questions were completed with two to three words, following the pattern of verb + object or verb + preposition + object. The object in the question always occurred in the sentence-final position to prevent early guessing of sentence meaning.

\section{Results}

Trials were excluded if the responses in the verification task were incongruent with the original responses or if response time was more than $3 S D$ s from the mean. This exclusion criterion eliminated 1 participant (incongruent responses exceeded $50 \%$ of total trials) and 92 trials (5\% of the data). Of the 1,908 trials remaining, 435 trials occurred in the false/no condition, 455 trials occurred in the true/no condition, 503 trials occurred in the false/yes condition, and 515 trials occurred in the true/yes condition.

Trajectory shape. With this analysis, we examined the shape of each trajectory as it moves from the bottom center bull's-eye to the final response box at the top left or top right of the screen. To conduct this analysis, we initialized the response trajectories for each participant to $x, y$ coordinates $(0,0)$ and interpolated to 101 time steps (see Dale et al., 2007; Spivey et al., 2005). At each time step, the $x$ - and $y$-coordinate positions were then averaged within conditions for each participant. To compare conditions, we performed paired $t$ tests at corresponding $x$-coordinate time steps (a total of $101 t$ tests). A consecutive run of statistically significant tests indicates that the trajectories between conditions diverged during response execution.

The false no and true no trajectories diverged for 29 time steps $(p<.05)$ between the 59th and 88th steps, whereas the false yes and true yes trajectories statistically diverged for 58 time steps $(p<.05)$ between the 40th and 98th steps (Figure 1A). The divergence for each comparison exceeds the minimum number of 8 consecutive time steps that bootstrap simulations have shown to be a standard for statistical significance (see Dale et al., 2007). Accordingly, the trajectory shape analysis revealed false and true responses that were conspicuously different. The true response movements appeared to travel a more direct route to the target response, whereas the false response movements took a more curved route. The bend of the curve was always in the direction of the competing response option (i.e., the true response). This greater curvature suggests that competition was greater for the false responses, whereas the processing for the true responses was relatively unaffected. 

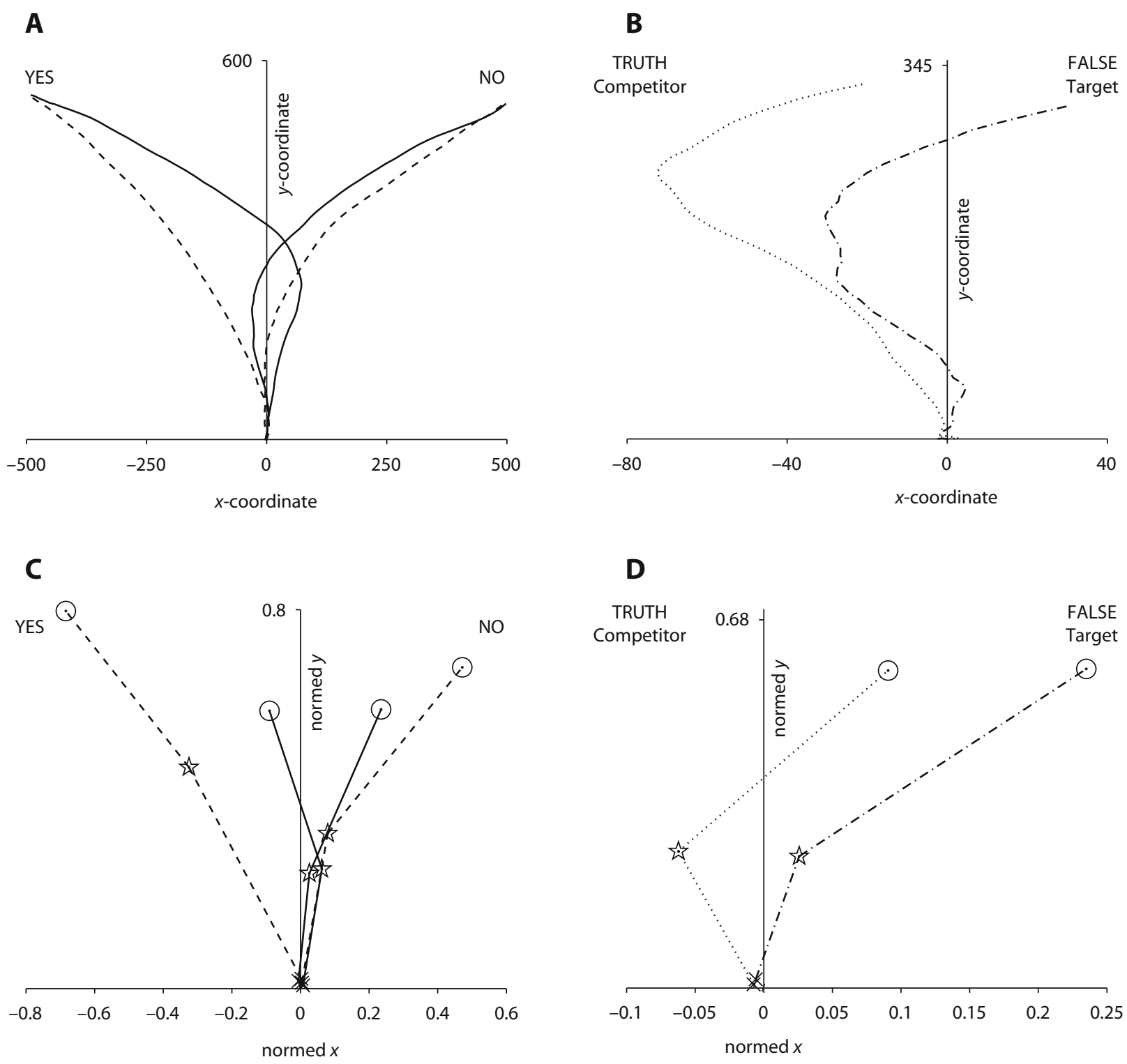

D

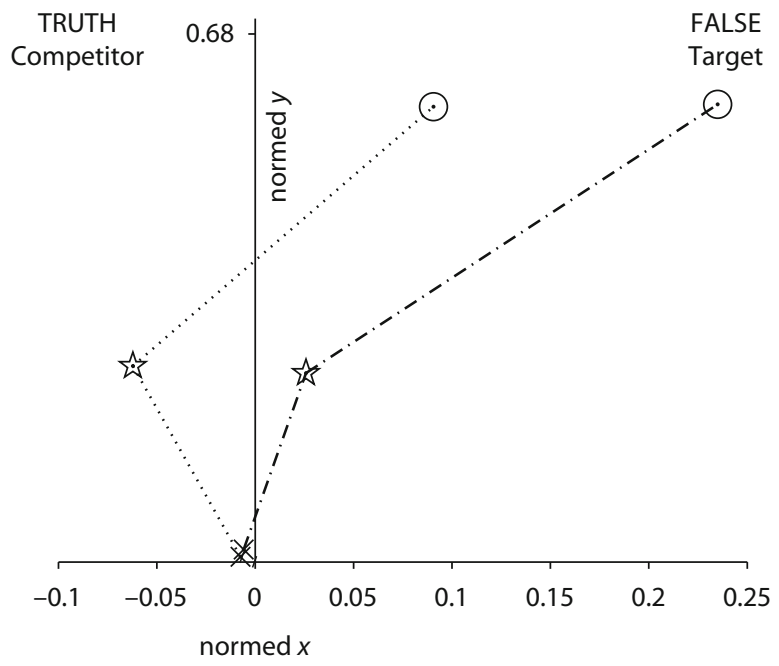

Figure 1. (A) Shape of Wii Remote trajectories for each condition. False answers (solid lines) display a greater arc toward the competing response option than do true answers (dashed lines). (B) Shape of trajectories for false yes responses (mirror reversed from trajectory in panel A) compared with the shape of false no responses. The false yes responses (dotted line) are closer to the competing true option than are the false $n o$ responses (dash-dotted line). (C) Location of Wii Remote trajectories for each condition. The $x$-coordinate position on each trajectory is plotted at $500 \mathrm{msec}$ (cross), 1,000 msec (star), and 1,500 msec (circle). The false answer positions (connected by solid lines) show slower movements toward the correct response location (e.g., the upper left corner for false yes) and are closer to the competing response option (e.g., the upper right corner for false yes) than do the true answers (connected by dashed lines). (D) Location of trajectories for false yes responses (mirror reversed from trajectory in panel B) compared with the shape of false no responses. The false yes responses (dotted line) are closer to the competing true option than are the false no responses (dash-dotted line).

To further explore this competition, we examined divergence between false no responses and false yes responses by superimposing mirror-reversed false yes trajectories (see Figure 1B). A paired $t$ test analysis revealed that the false yes responses diverged from the false no responses for 16 time steps $(p<.05)$ between the 55th and 70th time steps. During these time steps, the false yes responses were spatially closer to the competing true response option. Not only was there a greater competition for general false responding, but this effect was most pronounced with false yes responses.
Trajectory location. With this analysis, we compared the location of response trajectories after the first, second, and third $500 \mathrm{msec}$ of processing (for 500, 1,000, and $1,500 \mathrm{msec}$ ). This information was lost in the previous analysis when temporal information was collapsed into fixed time steps. Now with trajectory location, we can answer the question of when trajectories begin to be statistically divergent. To conduct this analysis, the trajectory coordinates were first normalized to initiate at $x, y$ coordinates $(0,0)$ and end at $(1,1)$. Next, the normalized 
$x$-coordinate positions for false and true responses were captured at the 500-, 1,000-, and 1,500-msec processing mark and placed in corresponding time bins. The average location of each time bin for each condition is plotted in Figure 1C.

A 2 (prompt type: true vs. false) $\times 3$ (time bin: 500 vs. 1,000 vs. 1,500 $\mathrm{msec}$ ) repeated measures ANOVA was used to evaluate trajectory position in real time. Beginning with the no trials, there were statistically significant effects for prompt type and time bin and a significant interaction between prompt type and time bin. To explore this interaction further, planned comparisons were conducted between prompt types at each time bin. There was a statistically significant difference of the $x$-coordinate position at the third time bin $(1,500 \mathrm{msec})$ between false $(M=0.23, S D=0.27)$ and true $(M=0.47, S D=0.23)$ trajectories $[F(1,24)=20.54, p<.001]$.

The repeated measures results for the yes trials also showed statistically significant effects for prompt type and time bin and a significant interaction between them. Planned comparisons for the interaction revealed statistically significant differences of the $x$-coordinate position at the second time bin $(1,000 \mathrm{msec})$ between false $(M=$ $0.06, S D=0.10)$ and true $(M=-0.31, S D=0.20)$ trajectories $[F(1,24)=81.40, p<.001]$ and at the third time bin $(1,500 \mathrm{msec})$ between false $(M=-0.09, S D=0.16)$ and true $(M=-0.68, S D=0.19)$ trajectories $[F(1,24)=$ 203.29, $p<.001]$.

Taken together, these results indicate that the false yes trajectories diverged from the true yes trajectories much earlier (at around 1,000 $\mathrm{msec}$ ) than when the false no trajectories began to diverge from the true no trajectories (not until at least $1,500 \mathrm{msec}$ ). Because divergence here refers to movement toward the competing response option, false yes trajectories appear to be influenced by a truth competition much earlier than do false no trajectories.

As in the trajectory shape analysis, false yes and no trajectories can be directly compared with each other instead of using the true trajectories as a reference, which have their own idiosyncratic response biases (as in a yes bias for no responses). As such, the trajectories for the false yes responses were mirror reversed (see Figure 1D), and paired $t$ tests at each time bin were assessed. Once again, there was greater divergence for false yes responses than for false no responses, with divergence recorded at the second time bin $[F(1,24)=4.31, p=.05]$ and at the third time bin $[F(1,24)=7.51, p=.01]$.

Trajectory velocity. The velocity of response trajectories was evaluated by computing the distance (in pixels) covered each second within a moving window of eight $x, y$ pixel coordinates across total time. Figure 2 shows the average velocity profile for each condition. This figure suggests that, on average, the initial increase in velocity (as the participants committed to a response) and the subsequent decrease in velocity (as the participants completed the response) occurred much later for false responses than for true responses. A repeated measures ANOVA conducted on the moment of peak velocity confirms this observation, such that a significant interaction $[F(1,24)=25.51$, $p<.001]$ and follow-up planned comparisons between

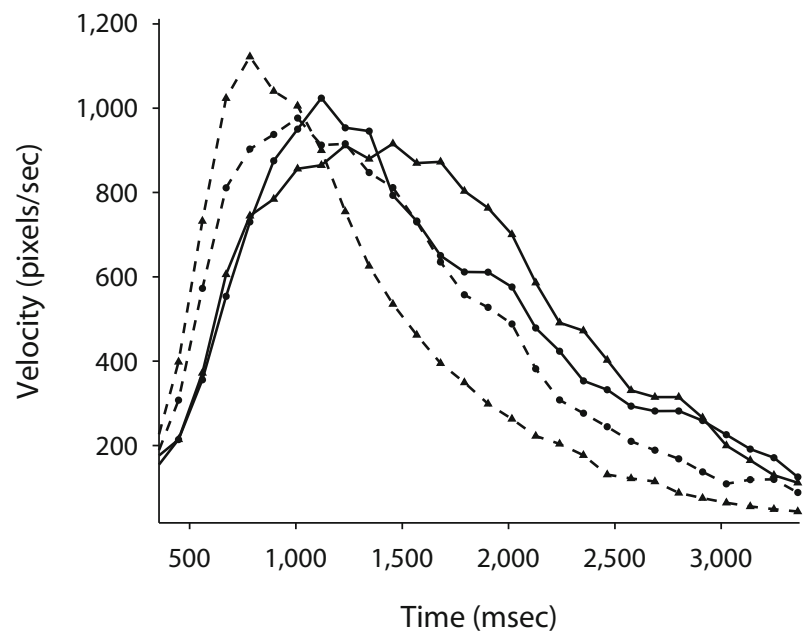

Figure 2. The velocity profiles plotted as a function of total time for true no (dashed line with circles), true yes (dashed line with triangles), false no (solid line with circles), and false yes (solid line with triangles) responses. The plotted range of 376-3,400 msec covers $90 \%$ of all completed trajectories and eliminates extremely early and late movements that have near-zero velocities. The average velocity profiles are defined over a broad range around the mean total time, because these profiles are partly based on slower trajectories that go beyond the mean total time- that is, trajectories with a total time that is longer than the mean total time.

prompt and response type reveal that false no responses peaked later than true no responses $[F(1,24)=22.29, p<$ $.001]$ and that false yes responses peaked later than true $y e s$ responses $[F(1,24)=93.32, p<.001]$.

We also examined differences in the magnitude of peak velocity with the assumption that greater response activation would result in higher peaks. A statistically significant interaction was found between prompt and response type $[F(1,24)=16.87, p<.001]$, showing that the peak for false yes responses is lower in magnitude than that of true yes responses $[F(1,24)=9.07, p<.01]$. There were no significant differences for false no and true no responses.

Trajectory properties. In this final analysis, we computed eight properties that characterize temporal and trajectory behavior along continuous scales of measurement (Dale et al., 2007). The variables are listed and summarized below.

Total time. The amount of time elapsed between the initiation of the prompt and making a yes or no response.

Latency. The amount of time that the mouse cursor stays in a latency region, with region defined as a 100pixel radius that surrounds the mouse cursor position that initiated the response prompt (Dale et al., 2008).

Distance. The Euclidean distance traveled by the trajectory after leaving the latency region and making a yes or no response.

Motion time. The amount of time elapsed between the cursor's moving between the latency region and the participants' completing a yes or no response.

High $x$ value. A measure of how close (in coordinate position) each trajectory curves toward the NO response box, with the No response box at the maximum $x$-value position. 
Table 1

Means and Standard Errors of the Wii Remote Trajectory Variables by Prompt and Response Type

\begin{tabular}{|c|c|c|c|c|c|c|c|c|}
\hline \multirow[b]{3}{*}{ Variable } & \multicolumn{4}{|c|}{ Yes } & \multicolumn{4}{|c|}{ No } \\
\hline & \multicolumn{2}{|c|}{ False } & \multicolumn{2}{|c|}{ True } & \multicolumn{2}{|c|}{ False } & \multicolumn{2}{|c|}{ True } \\
\hline & $M$ & $S E$ & $M$ & $S E$ & $M$ & $S E$ & $M$ & $S E$ \\
\hline Total time (msec) & 2,806 & 83 & 1,996 & 86 & 2,802 & 115 & 2,408 & 111 \\
\hline Latency (msec) & 1,247 & 73 & 999 & 59 & 1,246 & 67 & 1,111 & 57 \\
\hline Distance (pixels) & 1,423 & 102 & 996 & 98 & 1,426 & 145 & 1,273 & 114 \\
\hline Motion time (msec) & 1,558 & 103 & 997 & 65 & 1,556 & 122 & 1,297 & 107 \\
\hline High $x$ value & 229 & 24 & 69 & 12 & 515 & 4 & 515 & 4 \\
\hline Low $x$ value & -505 & 17 & -509 & 22 & -190 & 25 & -139 & 2 \\
\hline$x$ flips in latency & 8.67 & 0.66 & 6.87 & 0.50 & 9.00 & 0.78 & 8.32 & 0.79 \\
\hline$x$ flips in motion & 3.87 & 0.36 & 2.20 & 0.24 & 4.22 & 0.48 & 3.42 & 0.39 \\
\hline
\end{tabular}

Low $x$ value. A measure of how close (in coordinate position) each trajectory curves toward the YES response box, with the YES response box at the minimum $x$-value position.

$x$ flips in latency. The number of times that a trajectory moves back and forth on the $x$-axis within the latency region.

$x$ flips in motion. The number of times that a trajectory moves back and forth on the $x$-axis while in motion to a yes or no response.

The total time and latency variables are primarily temporal measures, whereas the remaining variables capture dynamical processes that occur along the trajectory of motion. For example, $x$ flips in latency and $x$ flips in motion provide an intuitive measure of response instability, and the high $x$ value and low $x$ value variables are indicators of competing attractor strengths that occur when the participants react either to $n o$ falsely (moving leftward on the $x$-axis toward the competing YES response region) or to yes falsely (e.g., moving rightward on the $x$-axis toward the competing NO response region)

A 2 (prompt type: false vs. true) $\times 2$ (response type: yes vs. no) repeated measures ANOVA was conducted for each of the eight dependent variables. Each variable, its mean value, and its standard error for each condition are provided in Table 1. The results of the repeated measures ANOVA are provided in Table 2.

To ensure that the results for trajectory properties were not unduly influenced by possible confounds, we also performed a hierarchical linear model (HLM) analysis

Table 2

$F$ Scores for the Repeated Measures Analysis Using Wii Remote Movements

\begin{tabular}{lccc}
\hline \multicolumn{1}{c}{ Variable } & $\begin{array}{c}\text { Yes Versus } \\
\text { No Response }\end{array}$ & $\begin{array}{c}\text { True Versus } \\
\text { False Prompt }\end{array}$ & $\begin{array}{c}\text { Prompt } \\
x \text { Response }\end{array}$ \\
\hline Total time (msec) & $9.92^{* *}$ & $80.67^{* *}$ & $11.85^{* *}$ \\
Latency (msec) & $4.68^{*}$ & $25.82^{* *}$ & \\
Distance (pixels) & $6.05^{*}$ & $16.03^{* *}$ & $4.70^{*}$ \\
Motion time (msec) & $8.04^{* *}$ & $44.12^{* *}$ & $6.90^{*}$ \\
High $x$ value & $693.63^{* *}$ & $68.38^{* *}$ & $72.72^{* *}$ \\
Low $x$ value & $288.49^{* *}$ & $7.18^{*}$ & $6.77^{*}$ \\
$x$ flips in latency & $5.09^{*}$ & $12.83^{* *}$ & \\
$x$ flips in motion & $9.41^{* *}$ & $38.50^{* *}$ & \\
\hline Tote &
\end{tabular}

Note-The degrees of freedom for all analyses were 1,24. ${ }^{*} p<$ .05 . ${ }^{* *} p<.001$. with random factors that controlled for variance due to practice effects (by nesting trial number in participants), for $\mathrm{NO} /$ YES response position, and for items. A noted advantage of an HLM analysis is that it is also quite robust against unequal $n \mathrm{~s}$ in conditions, as is the case with our data. In all analyses, the results were consistent with the repeated measures ANOVA, except that the interaction terms for latency and $x$ flips in latency were no longer significant.

\section{DISCUSSION}

The present study is the first investigation into the action dynamics of deceptive behavior. The movements of the arm revealed distinct signatures of cognitive activity as the participants made false and true responses to autobiographical questions. During false responses, the dynamics were slower and more disorderly than those during true responses, and they were also curved toward a competitor true region that was visually copresent with the target response region. This curvature suggests the presence of a truth-bias attractor that pulls processing off course during the production of a false response (Gilbert et al., 1993; McKinstry, Dale, \& Spivey, 2008). For truthful responses, there was no equivalent pull in the direction of a competitor false region.

The competition effects for false responding are similar to those in decision tasks that involve competition between featurally similar exemplars (Dale et al., 2007), ambiguous syntactic completions (Farmer, Cargill, Hindy, Dale, \& Spivey, 2007), and phonological competitors (Spivey et al., 2005). As in these studies, the competition exhibited in false responding is the result of cognitive components that evolve smoothly over the response movement itself, suggesting overlapping processes in overcoming an initial belief and generating a false response. This view of deception naturally extends response time measures by incorporating the fine-grained changes that occur during the response. By doing so, a clearer distinction between deceptive and truthful behavior is possible.

One notable distinction is the greater trajectory curvature and slower responses for false yes responses than for false no responses. This differentiation within the false responses was not found for the truth responses. Interestingly, the greater difficulty of falsely responding yes 
is at odds with an earlier finding that normal yes responses elicit faster and smoother trajectories than do no responses - a yes bias (McKinstry et al., 2008). Indeed, on the basis of Gilbert and colleagues' (Gilbert, 1991; Gilbert et al., 1993) findings, automatic acceptance of propositions should give yes responses a facilitative advantage. However, when deception is involved, this yes automaticity conflicts with the more deliberative goal to respond falsely.

To lay the initial foundation in quantifying deceptive response movements, we chose a guided lie paradigm that generally permits a straightforward contrast between false and true response behaviors. Unfortunately, this distinction is not always realized in real-world scenarios, in which elements of truth are intermingled with the motives and content of a lie. There is also a limitation in our experimental paradigm that concerns the intention to deceive. Ekman (1997) argued that deception is an act of conscious volition that requires the deceiver to know what is accurate and then to purposefully violate that knowledge with false information. Clearly, deception requires a certain degree of motivation that is absent from a guided lie paradigm. Nevertheless, this type of responding is still closely aligned with deceptive behavior and is widely used and accepted in the deception literature (DePaulo et al., 2003; Vrij, 2001). Of course, consensus does not negate further investigation, and future work will allow participants greater choice in the scope of their deception, both in when they deceive and under what circumstances they do it (e.g., personal or social gain, avoiding embarrassment).

It is clear that deception is a complex behavior that garners both theoretical and applied attention. To concoct a false reality requires one to maintain a mental representation of the truth and then to violate this representation with all appearances of sincerity. Not only is this behavior cognitively challenging, but it also interacts with a host of social, motivational, and emotional factors. Notwithstanding this complexity, researchers have devised a multitude of techniques to identify cues of deception. This study provides the first steps toward applying an action dynamics framework to the exploration of false response behavior. The results suggest that dynamic measures capture deceptive processes that are unavailable to response time measurements alone. If so, these measures could improve existing prediction models that have been touted in recent years (Gregg, 2007; Sartori et al., 2008; Walczyk, Mahoney, Doverspike, \& Griffith-Ross, 2009), as well as supplement techniques for detecting online deception (Monrose \& Rubin, 2000). Although there is much more work ahead, we admonish deceivers everywhere: Your arm might just reveal when you are lying.

\section{AUTHOR NOTE}

This research was supported by a National Science Foundation (NSF) Graduate Research Fellowship to the first author, by NSF Grant BCS0720322, and by IES Grant R305a080589. Correspondence concerning this article should be addressed to N. D. Duran, Department of Psychology, University of Memphis, 202 Psychology Building, Memphis, TN 38152-3230 (e-mail: nduran@memphis.edu).

\section{REFERENCES}

Dale, R., Kehoe, C., \& Spivey, M. J. (2007). Graded motor responses in the time course of categorizing atypical exemplars. Memory \& Cognition, 35, 15-28.

Dale, R., Roche, J., Snyder, K., \& McCall, R. (2008). Exploring action dynamics as an index of paired-associate learning. PLOS ONE, 3, e1728. doi:10.1371/journal.pone.0001728.

DePaulo, B. M., Lindsay, J. J., Malone, B. E., Muhlenbruck, L., Charlton, K., \& Cooper, H. (2003). Cues to deception. Psychological Bulletin, 129, 74-118.

EkMAN, P. (1997). Lying and deception. In N. L. Stein, P. A. Ornstein, B. Tversky, \& C. Brainerd (Eds.), Memory for everyday and emotional events (pp. 333-348). Mahwah, NJ: Erlbaum.

Farmer, T. A., Cargill, S. A., Hindy, N. C., Dale, R., \& Spivey, M. J. (2007). Tracking the continuity of language comprehension: Computer mouse trajectories suggest parallel syntactic processing. Cognitive Science, 31, 889-909.

Gilbert, D. T. (1991). How mental systems believe. American Psychologist, 46, 107-119.

Gilbert, D. T., Tafarodi, R. W., \& Malone, P. S. (1993). You can't not believe everything you read. Journal of Personality \& Social Psychology, 65, 221-233.

GREGG, A. P. (2007). When vying reveals lying: The timed antagonistic response alethiometer. Applied Cognitive Psychology, 21, 621-647.

Just, M. A., Carpenter, P. A., \& Woolley, J. D. (1982). Paradigms and processes in reading comprehension. Journal of Experimental Psychology: General, 111, 228-238.

McKinstry, C., Dale, R., \& Spivey, M. J. (2008). Action dynamics reveal parallel competition in decision making. Psychological Science, 19, 22-24.

Monrose, F., \& Rubin, A. D. (2000). Keystroke dynamics as a biometric for authentication. Future Generation Computer Systems, 16, 351-359.

Sartori, G., Agosta, S., Zogmaister, C., Ferrara, S. D., \& Castiello, U. (2008). How to accurately assess autobiographical events. Psychological Science, 19, 772-780.

Spence, S. A., Hunter, M. D., Farrow, T. F. D., Green, R. D., Leung, D. H., Hughes, C. J., \& Venkatasubramanian, G. (2004). A cognitive neurobiological account of deception: Evidence from functional neuroimaging. Philosophical Transactions of the Royal Society B, 359, 1755-1762.

Spivey, M. J., Grosjean, M., \& Knoblich, G. (2005). Continuous attraction toward phonological competitors. Proceedings of the National Academy of Sciences, 102, 10393-10398.

Vendemia, J. M., \& BuZAN, R. F. (2004). Neuronal mechanisms of deception and response congruity in a visual two-stimulus paradigm involving autobiographical information. Psychophysiology, 40, 25-36.

VRIJ, A. (2001). Detecting lies and deceit: The psychology of lying and the implications for professional practice. Chichester, U.K.: Wiley.

Walczyk, J. J., Mahoney, K. T., Doverspike, D., \& Griffith-Ross, D. A. (2009). Cognitive lie detection: Response time and consistency of answers as cues to deception. Journal of Business \& Psychology, 24, 33-49.

Walczyk, J. J., Schwartz, J. P., Clifton, R., Adams, B., Wei, M., \& ZHA, P. (2005). Lying person-to-person about life events: A cognitive framework for lie detection. Personnel Psychology, 58, 141-170.

\section{NOTE}

1. http://actiondeception.nickduran.com/, as well as in the supplemental materials.

\section{SUPPLEMENTAL MATERIALS}

Supplemental figures depicting the apparatus and a sample trial sequence, as well as a list of the sentence stimuli, may be downloaded from http://pbr.psychonomic-journals.org/content/supplemental.

(Manuscript received August 5, 2009; revision accepted for publication February 17, 2010.) 